\title{
Heyer, Andreas (2019): Die Verfassung der Jakobiner von 1793 und ihr historischer Kontext
}

\author{
Baden-Baden: Nomos. 313 Seiten. $59 €$
}

\author{
Axel Rüdiger
}

Angenommen: 21. Dezember 2020 / Online publiziert: 11. Januar 2021

(C) Der/die Autor(en) 2021

Die Zukunft der Demokratie dürfte unter der gegebenen desaströsen global-kapitalistischen Dynamik wohl keiner hedonistisch-diversen Marktutopie, sondern bestenfalls einer kollektiv und global organisierten Rettungsaktion gleichen, deren riskante Dramatik am ehesten aus großen Grubenunglücken bekannt ist. So zumindest lautet die ernste Prognose, wie sie von Terry Eagleton und Frederic Jameson vertreten wird. Aus der Perspektive der Politischen Theorie und Ideengeschichte lässt sich diese Vorstellung durchaus auf reale historische Vorgänge zurückführen, deren ursprünglich territorial begrenzte Problematik nun allerdings auf globaler Ebene zurückkehrt. Schon die politische Gründung des demokratischen Republikanismus als verfasste Staatsform in der jakobinischen Phase der Französischen Revolution zwischen 1792 und 1794 lässt sich nach einem solchen Rettungsmuster deuten. Dies wird bei der Lektüre der Monografie von Andreas Heyer über die jakobinische Verfassung von 1793 sehr deutlich, was dem Text über die rein historische Rekonstruktion der demokratisch-republikanischen Verfassungstradition hinaus eine besondere politische Aktualität verleiht.

Der Autor stellt sich mit engagierter Verve dem grassierenden historisch-politischen Revisionismus entgegen, der ausgehend von der konservativ-liberalen Schule François Furets und Claude Leforts schließlich vom antijakobinischen Furor des „progressiven Neoliberalismus“ (Nancy Fraser) vollendet wurde. In einer längeren historischen Einleitung (90 Seiten) wird die revisionistische Umkehrung der historisch-politischen Kausalität, in der ausgerechnet und ausschließlich die Gründung der demokratischen Republik zur katastrophalen Entgleisung der Revolution - mit tendenziell totalitären Folgen - gerät, am historischen Detail zurückgewiesen. Der Fokus liegt hierbei neben der revolutionären Verfassungsdebatte zwischen 1789 und

A. Rüdiger $(\bowtie)$

Martin-Luther-Universität Halle-Wittenberg, Halle/Saale, Deutschland

E-Mail: axel.ruediger@politik.uni-halle.de 
dem Sturz der Monarchie am 10. August 1792 insbesondere auf der für die Revolution entscheidenden Frage über Krieg und Frieden. Deutlich wird hierbei, wie sich der radikale Flügel der Jakobiner um die „Bergpartei“ Maximilien Robespierres der bellizistischen Eskalationsstrategie, die sowohl von den Monarchisten als auch vom Großbürgertum mit jeweils eigenen Absichten betrieben wurde - vor allem aber, um die soziale Dynamik der Revolution ruhig zu stellen -, energisch widersetzte.

Ergänzen ließe sich diese wichtige Richtigstellung auch durch die von Edmund Burke schon 1790 angeprangerte liberale Katastrophenpolitik bezüglich der Finanzierung der Staatsschulden zugunsten der Gläubiger. Über die Berücksichtigung der ökonomischen Hintergründe der Revolution hinaus, die im Buch leider nur sehr abstrakt vorkommt, kann damit auch die revisionistische und zumeist als selbstverständlich vorgetragene Bezugnahme auf Burke als Kritiker der Französischen Revolution effektiv infrage gestellt werden. Denn Burke zufolge war die Politik der Nationalversammlung von 1789 keineswegs die Lösung der Finanzprobleme des Ancien Régime, sondern nur ein weiterer Schritt zu auf den politisch-sozialen Abgrund, über den nach äußeren und inneren Krieg sowie einer Hungerkrise allein ein Weg aus „Feuer und Blut“ hinausführt. Entgegen der etablierten politischen Romantik, die beim Datum von 1789 von einem makellosen Triumph der Freiheit ausgeht, der erst vom Sturz der Monarchie und der Gründung der demokratischen Republik beschmutzt wird, sieht der realpolitische Blick die Katastrophe vom Anfang und nicht vom Ende her.

Im Hauptteil der Monografie diskutiert Heyer in verschiedenen Kapiteln die komplizierte Genese der ersten republikanischen Verfassung, die nach dem Sturz der Monarchie die politisch überholte Verfassung von 1791 ersetzen sollte. Detailliert beschrieben werden die Auseinandersetzungen im republikanischen Konvent 1792/93 vor dem Hintergrund der Spaltung des Jakobinerklubs (ab 1791 offiziell „Gesellschaft der Verfassungsfreunde“) in die im Oktober 1792 ausgeschlossenen liberalen „Girondisten“ und die radikaldemokratische „Bergpartei“ (,Montagne“). Im Vordergrund steht hierbei die Entdämonisierung der ,,vermeintlichen Ungeheuer und schlimmsten Verbrecher der Revolution“ (,Pétion, Marat, natürlich Robespierre“) (S. 76), die sich in den Worten von Louis Antoine Saint-Just als legitime „Deputierte der Menschheit und der Republik“ (S. 76) zur Rettung der Revolution den „kleinen Freuden der Bourgeoisie“ (S. 65) wie Krieg und Spekulation entgegenstellten. Die noch von der Gironde im neu gewählten republikanischen Konvent angestoßene Verfassungsdebatte wird in ihren verschiedenen Etappen unter der besonderen Berücksichtigung der Beiträge von Marie Jean Antoine Nicolas de Caritat, Marquis de Condorcet, Robespierre, Saint-Just und Marie Jean Hérault de Séchelles breit dargestellt und interpretiert. Dabei widmet sich der Autor in zwei Kapiteln Condorcet, dessen politische Philosophie zunächst nach kontraktualistischen und utopischen Elementen befragt wird, um anschließend den Verfassungsentwurf des Marquis zu würdigen. Obwohl Condorcet trotz seines girondistischen Mandates eine gewisse politische Unabhängigkeit bescheinigt wird, werden die demokratischen Tendenzen seines Entwurfs, die insbesondere in der Forderung nach politischer Gleichberechtigung der Frauen zum Ausdruck kommen, aufgrund seiner besitzindividualistischen Grundorientierung als kulturelle „Alibi-Funktion“ (S. 168) im Dienste bürgerlicher „Klassenpolitik“ (S. 167) bewertet. Die hierüber geführte 
Polemik mit Daniel Schulz ist allerdings eine der wenigen Auseinandersetzungen mit der neueren Forschungsliteratur, deren pauschale Abwertung en bloc gerade deshalb nicht restlos zu überzeugen vermag.

Nach der Darstellung von Robespierres politischer Ideenwelt im Umfeld des Sturzes der Gironde und des finalen Verfassungsentwurfs von Hérault de Séchelles kommen auch linke Kritiker wie Jacques Roux und François-Joseph L'Ange zu Wort, denen der Entwurf vom Juni 1793, etwa in der Bekämpfung des Spekulantentums, nicht weit genug ging. Letztlich artikuliert sich laut Heyer in der Verfassung vom 24. Juni 1793 ein politischer Kompromiss zwischen der siegreichen Bergpartei und der geschlagenen Gironde, der auf demokratische Weise alle republikanischen Kräfte zur Rettung der revolutionären Nation in einer militärisch nahezu aussichtslosen Situation mobilisieren sollte.

Der von Heyer herausgearbeitete innovative Charakter der Verfassung lässt sich folgendermaßen zusammenfassen: Das demokratische Prinzip der Volkssouveränität bleibt nicht allein auf eine juristische Fiktion beschränkt, sondern bildet über die Anerkennung der konstituierenden Macht des politischen Widerstandes gegen Unterdrückung eine reale praktische Größe innerhalb der politischen Ordnung. Das jakobinische Verbot der kolonialen Sklaverei war die logische Folge. Ferner wird das allgemeine Wahlrecht aller volljährigen, obgleich nur männlichen Bürger mit der plebiszitären Bestätigung der Gesetzgebung kombiniert, was dazu geeignet ist, den oligarchischen Effekt politischer Entfremdung per Delegation demokratisch effektiv zu beschränken. Gleichzeitig wird die Unverletzlichkeit des freien Mandats garantiert und mit einer meritokratischen Verpflichtung auf das Gemeinwohl verknüpft; was nebenbei gesagt ganz im Sinne Burkes ist. Das Recht auf Arbeit, die Ächtung des Angriffskrieges, unbeschränktes politisches Asyl sowie freie Religionsausübung runden die bis in das 20. Jahrhundert hinein fortschrittlichste Verfassung überhaupt $\mathrm{ab}$.

Dass die jakobinische Verfassung niemals in Kraft getreten ist, verdankt sich zunächst dem Dekret über die Revolutionsregierung, welches die Konstitution aufgrund der Kriegslage bis zum Frieden zugunsten der Diktatur des Wohlfahrtsausschusses suspendierte. Auf den anschließenden Terror geht das Buch nur kurz ein, wobei der Legitimationsverlust, der zum Sturz der Robespierristen im August 1794 führte, auf die Verweigerung einer ,sansculottischen Wirtschaftspolitik“ (S. 282) zugunsten einer dirigistischen Preispolitik für Lebensmittel zurückgeführt wird. Die Pervertierung des Terrors liegt demnach strukturell im Zurückschrecken vor einer ,radikalsozialen Dimension der Demokratie“ (S. 282) begründet. Nach dem Sieg der davon profitierenden Thermidorianer wurde die Propagierung der jakobinischen Verfassung allerdings bald unter Todesstrafe gestellt, die François Noël Babeuf 1797, nachdem er die Verfassung mithilfe einer politischen Verschwörung doch noch in Kraft zu setzen trachtete, tatsächlich ereilte. Mit der Kritik Babeufs an der besitzbürgerlichen Neudefinition der „Demokratie“ in der Verfassung von 1795 endet die Monografie über jene Verfassung, die am institutionellen Anfang des modernen demokratischen Republikanismus steht, aber im Zuge der besitzbürgerlich-liberalen Aneignung des Demokratiediskurses mit Hilfe der Totalitarismusdoktrin weitgehend aus dem kollektiven Gedächtnis getilgt wurde. 
Die große Stärke der Monografie besteht darin, noch einmal die ,klassisch(en) soziale(n) Interpretation der Französischen Revolution“ (S. 280), angefangen von Jules Michelet, über Jean Jaurès, Albert Mathiez, Georges Lefebvre bis hin zu Albert Soboul, Walter Markov und Walter Grab, um nur einige Namen zu nennen, gegen den aktuellen Revisionismus zu Wort kommen zu lassen. Die revisionistische Bevorzugung der konstitutionellen Monarchie oder der Girondisten als Ahnen eines vermeintlich gar radikalen Demokratiediskurses wird energisch zurückgewiesen. Zum Zweck der „Beschämung der Forschung der letzten Jahrzehnte“ (S. 270), in der sich bestenfalls „,die wissenschaftliche Klassenpolitik der Bourgeoisie“ (S. 271) niederschlägt, wird oft und ausführlich aus den klassischen Werken der Revolutionshistoriografie zitiert.

Die kraftvolle, aber mitunter in die Grauzone des Feuilletonismus abgleitende Polemik des Autors tut sich und seiner Sache jedoch keinen wirklichen Gefallen, wenn sie neben dem mitunter problematischen Stil bis auf wenige Ausnahmen auch nahezu komplett auf die Forschungsliteratur der letzten 30 Jahre zum Republikanismus in der Französischen Revolution verzichtet. Diese lässt sich nämlich keinesfalls restlos unter das Stigma des Revisionismus subsumieren. Namen wie etwa Minchul Kim, Marisa Linton, John P. McCormick, Michael Sonenscher oder Sophie Wahnich sind im Literaturverzeichnis leider nicht zu finden. Die vom Rezensenten selbst vor einiger Zeit geforderte Fortschreibung der republikanischen Revolutionsgeschichte unter den wissenschaftlichen Bedingungen des 21. Jahrhunderts bleibt daher weiterhin offen. Dennoch erwirbt sich das Buch große Verdienste bei der Entmystifizierung des jakobinischen Gründungsaktes des demokratischen Republikanismus und ist daher nicht nur den Leserinnen des sozialistischen Politik-Magazins ,Jacobin“ zu empfehlen, dessen Logo mit dem Konterfei von Toussaint Louverture ein schwarzer Jakobiner ziert, woraus abzulesen ist, dass es hier nicht nur um Europas Zukunft geht. Vielleicht hält diese Zukunft also auch einen politischen ,Fortschritt in dunklen Zeiten“ (Markus Gabriel) bereit?

Funding Open Access funding enabled and organized by Projekt DEAL.

Open Access Dieser Artikel wird unter der Creative Commons Namensnennung 4.0 International Lizenz veröffentlicht, welche die Nutzung, Vervielfältigung, Bearbeitung, Verbreitung und Wiedergabe in jeglichem Medium und Format erlaubt, sofern Sie den/die ursprünglichen Autor(en) und die Quelle ordnungsgemäß nennen, einen Link zur Creative Commons Lizenz beifügen und angeben, ob Änderungen vorgenommen wurden.

Die in diesem Artikel enthaltenen Bilder und sonstiges Drittmaterial unterliegen ebenfalls der genannten Creative Commons Lizenz, sofern sich aus der Abbildungslegende nichts anderes ergibt. Sofern das betreffende Material nicht unter der genannten Creative Commons Lizenz steht und die betreffende Handlung nicht nach gesetzlichen Vorschriften erlaubt ist, ist für die oben aufgeführten Weiterverwendungen des Materials die Einwilligung des jeweiligen Rechteinhabers einzuholen.

Weitere Details zur Lizenz entnehmen Sie bitte der Lizenzinformation auf http://creativecommons.org/ licenses/by/4.0/deed.de. 